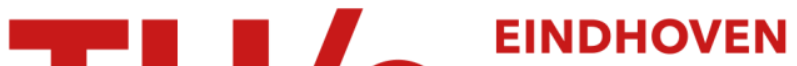 UNIVERSITY OF TECHNOLOGY
}

\section{Theoretical analysis of a feedback insensitive semiconductor ring laser using weak intracavity isolation}

Citation for published version (APA):

van Schaijk, T. T. M., Lenstra, D., Bente, E. A. J. M., \& Williams, K. A. (2018). Theoretical analysis of a feedback insensitive semiconductor ring laser using weak intracavity isolation. IEEE Journal of Selected Topics in Quantum Electronics, 24(1), [1800108]. https://doi.org/10.1109/JSTQE.2017.2720966

DOI:

10.1109/JSTQE.2017.2720966

Document status and date:

Published: 01/01/2018

Document Version:

Accepted manuscript including changes made at the peer-review stage

Please check the document version of this publication:

- A submitted manuscript is the version of the article upon submission and before peer-review. There can be important differences between the submitted version and the official published version of record. People interested in the research are advised to contact the author for the final version of the publication, or visit the $\mathrm{DOI}$ to the publisher's website.

- The final author version and the galley proof are versions of the publication after peer review.

- The final published version features the final layout of the paper including the volume, issue and page numbers.

Link to publication

\section{General rights}

Copyright and moral rights for the publications made accessible in the public portal are retained by the authors and/or other copyright owners and it is a condition of accessing publications that users recognise and abide by the legal requirements associated with these rights.

- Users may download and print one copy of any publication from the public portal for the purpose of private study or research.

- You may not further distribute the material or use it for any profit-making activity or commercial gain

- You may freely distribute the URL identifying the publication in the public portal.

If the publication is distributed under the terms of Article 25fa of the Dutch Copyright Act, indicated by the "Taverne" license above, please follow below link for the End User Agreement:

www.tue.nl/taverne

Take down policy

If you believe that this document breaches copyright please contact us at:

openaccess@tue.nl

providing details and we will investigate your claim. 


\title{
Theoretical analysis of a feedback insensitive semiconductor ring laser using weak intracavity isolation
}

\author{
T. T. M. van Schaijk, Member, IEEE, D. Lenstra, Senior Member, IEEE, E. A. J. M. Bente, Member, IEEE \\ and K. A. Williams, Member, IEEE
}

\begin{abstract}
External optical feedback can severely deteriorate the performance of semiconductor lasers. This work proposes an integrated laser design that can withstand tens of percent of offchip feedback, without requiring the integration of magneto-optic materials. The proposed laser consists of a ring cavity with a weak intracavity optical isolator. Sufficient gain difference between clockwise and counter-clockwise modes leads to unidirectional laser oscillation. Any reflected light is returned to a mode that is below threshold. This significantly reduces interactions between the feedback and the lasing mode. A rate-equation analysis is presented to show that the relative intensity noise changes less than a factor 2 when less than $-0.1 \mathrm{~dB}$ of the light is fed back into the laser and when intracavity isolation is $10 \mathrm{~dB}$. Linewidth and optical output power change approximately $1 \%$ for these values.
\end{abstract}

Index Terms-Ring lasers, Semiconductor lasers, Optical feedback, Photonic integrated circuits, Rate equation modelling

\section{INTRODUCTION}

$\mathbf{S}$ EMICONDUCTOR lasers can be particularly sensitive to external optical feedback (EOF), as was recognized long ago [1]-[4]. Five feedback regimes have been identified in a distributed feedback laser based on the feedback power ratio and the distance to the reflection [1]. The distance to the reflection only plays a role for ratios below $-50 \mathrm{~dB}$. Going from low to high reflection, feedback can cause external cavity modes, line narrowing, extreme line broadening and finally stabilization when the laser field is dominated by the feedback. In many circumstances the strength of the reflection is unpredictable and can fluctuate over time, resulting in an undesirable change in the output laser light. To solve this problem, one or often two Faraday isolators are employed to prevent EOF from returning to the laser cavity altogether [5]-[8]. From [1] it is found that feedback power ratios below $-60 \mathrm{~dB}$ have no observable effect on the lasing mode of a distributed feedback laser for typical feedback distances.

Attempts to obtain Faraday isolation on chip have been made [9]-[19]. So far the insertion loss and integration complexity have proven a barrier to implementation of nonreciprocal elements however. Recently a class of isolators has

This work is part of the research programme Memphis 2 with project number 13540, which is (partly) financed by the Netherlands Organisation for Scientific Research (NWO).

The authors are with the Institute of Photonic Integration (formerly the COBRA institute), Eindhoven University of Technology, Eindhoven, The Netherlands (email: p.v.schaijk@tue.nl; d.lenstra@tue.nl; e.a.j.m.bente@tue.nl; k.a.williams@tue.nl). been devised which avoids the use of magneto-optic materials altogether. It was found that isolation can be achieved using non-linearities [20], but this type of isolator can only be used as when light is either propagating in the forward or the reverse direction. These devices are therefore not suitable for preventing the detrimental effects of EOF on continuous wave lasers, but can be used for pulsed lasers as was proposed in [21]. Isolation can also be obtained using a time varying refractive index [22] [25], but none of these have achieved the $60 \mathrm{~dB}$ required to fully suppress the effects of EOF.

This work takes a different approach to suppressing the effects of EOF on a continuous wave, single frequency, tunable laser. The principle is to allow light to get back into the laser cavity, but into a different, sub-threshold mode. This is achieved by placing a weak isolator inside the cavity of a ring laser such that one direction is favoured over the other. E.g. the clockwise (cw) mode reaches threshold and is lasing, while EOF returns to the counterclockwise (ccw) mode. With a gain difference of only $0.5 \mathrm{~dB}$ the ccw mode is suppressed by approximately $40 \mathrm{~dB}$ relative to the $\mathrm{cw}$ mode [26]. For large external reflection, EOF contributes significantly to the power in the ccw mode and larger isolation is required, at most $10 \mathrm{~dB}$ as will be shown later. It will be shown that by using a weak intracavity isolator the effects of EOF on the lasing mode can be greatly reduced. This is in stark contrast to a ring laser that is forced to oscillate unidirectionally using an external mirror, which was studied using a rate equation analysis in [27]. As was shown in [25], it is possible to achieve the required isolation using InP based waveguides and phase modulators but the principle holds for any integration platform that is able to create $10 \mathrm{~dB}$ of isolation. A less detailed study of the steady state behaviour of this laser was already performed by us in [28], while [29] studied the dynamic behaviour of the laser in some detail.

In this paper we will analyze an integrated ring laser with intracavity isolator in detail to obtain a good insight into the performance of the laser when it is subjected to EOF and to determine the requirements on the isolator. Section II first gives an intuitive explanation why a unidirectional ring laser with weak intracavity isolator will be less susceptible to EOF. Section III then goes on to describe the model that is used to analyze the laser providing the assumptions which have been made. Section IV details the analysis of the laser for the steady state, resulting in the average values of the optical intensity and number of charge carriers, and section $\mathrm{V}$ introduces the effects of spontaneous emission noise. As a result of this analysis 


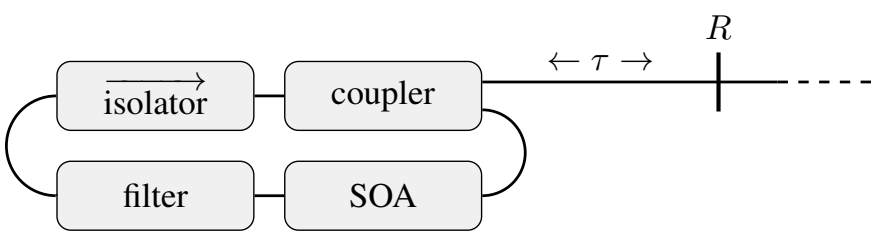

Figure 1. Schematic overview of a feedback insensitive laser subjected to EOF. The laser consists of a ring cavity and is assumed to be lasing the $\mathrm{cw}$ direction. The coupler couples some of the light to the outside world and the SOA acts as an amplifier. Lasing in a single longitudinal mode is ensured by the filter and by the cavity itself, while the isolator ensures unidirectional lasing. EOF is modeled by a point-reflection that reflects a fraction $R$ of the optical power. $\tau$ models the feedback delay time.

the relative intensity noise (RIN) spectrum and linewidth are obtained as a function of the feedback rate and intracavity isolation. These values are used to quantify the performance of the laser. Finally, section VI concludes the paper.

\section{CONCEPT OF LASER OPERATION}

Ring lasers can have the special property that light that is reflected back into the output port of the laser couples to a different mode than the emitting mode if the two modes are not optically coupled inside the cavity. If the laser is for example lasing in a mode that is propagating in the $\mathrm{cw}$ direction, light that is reflected at the output of the laser will couple into the ccw mode of the laser. If there is no backscattering present, there will be no interference between the emitting mode and the light from the feedback. This means the laser does not exhibit external cavity modes. This type of laser therefore does not suffer from mode hopping and other destabilizing effects typically caused by the presence of external cavity modes.

The EOF, coupling into the $\mathrm{ccw}$ mode, does still affect the carrier concentration in the semiconductor optical amplifier (SOA). This may cause a small coupling between the two modes. However, EOF will have a much smaller effect on the emitting mode and thus on the output of the laser, if the ccw mode can be kept below the threshold for lasing. This gain difference can be ensured by integrating an optical isolator into the laser cavity. Such an isolator only has to provide a small loss difference between the two propagation directions, which will ensure that the laser prefers one over the other. The required isolation is much smaller than the isolation that would be required by an external isolator.

Our proposed laser architecture is schematically shown in Fig. 1. It includes an SOA to provide amplification, a spectral filter to ensure that only a single longitudinal mode per direction can have sufficient gain to reach threshold, an isolator to suppress the light in one of the propagation directions and a coupler to couple light out of the cavity. The interaction between the cw and ccw modes is expected to occur in the SOA only, requiring any waveguide transitions to have sufficiently low reflectivity.

\section{MODEL DESCRIPTION}

To model the laser under study, a set of rate equations is formulated. This allows a study of the dynamical behavior of the laser, including the response induced by EOF. Because of the intracavity filter the laser is single mode in both directions and the rate equations that need to be considered can be simplified significantly. Since the $\mathrm{cw}$ and $\mathrm{ccw}$ modes have equal wavelength, they both interact with charge carriers of the same energy level in the SOA. Thus, the laser can be described using a set of three rate equations: two for the complex optical fields in both the $\mathrm{cw}$ and $\mathrm{ccw}$ modes and one for the number of charge carriers.

It is assumed that the number of carriers and the intensities and frequencies will reach a stable average value after some time - the steady state - after which only minor excursions from these values occur due to spontaneous emission noise. Since only small excursions are assumed, all effects are linearized around the steady state to achieve considerable simplification in describing the effect of noise. It is assumed that all the EOF originates from a single point of reflection outside of the laser cavity, indicated by $R$ in Fig. 1. For a photonic integrated circuit (PIC) this can for instance be a reflection of a fiberconnector. Because only a single point of reflection is assumed, use can be made of the equations derived in the pioneering paper by Lang and Kobayashi [30].

This study builds further on the ideas of [30], and extends these by including the loss difference induced by the isolator and obtaining a model for a ring laser. It is assumed that the only coupling between the $\mathrm{cw}$ and $\mathrm{ccw}$ modes is through the EOF and the changes in carrier density. Effects such as backscattering in the waveguides are assumed to be negligible. The isolator is modeled as a loss difference between the $\mathrm{cw}$ and cew modes.

\section{RATE EQUATIONS}

To obtain the steady state intensities and frequencies of both modes as well as the number of charge carriers at steady state, this section first formulates a fully deterministic model that does not include EOF. Only after this model is completed, feedback effects are added to the model. Finally stochastic terms which model the influence of spontaneous emission are added. This treatment greatly simplifies the analysis and was done for linear lasers in [26].

The complex electric field strength of the $\mathrm{cw}$ and $\mathrm{ccw}$ modes and the number of carriers shall be indicated with $\mathcal{E}_{\mathrm{cw}}, \mathcal{E}_{\mathrm{ccw}}$ and $n$ respectively. $\mathcal{E}_{c w}$ is normalized such that $\left|\mathcal{E}_{\mathrm{cw}}\right|^{2}$ is equal to the number of photons in the cw mode in the cavity, or more correctly the energy in the cw mode divided by the energy per field quantum, $\hbar \omega$, where $\omega$ is the optical angular frequency of the mode. A similar scaling is performed for $\mathcal{E}_{\text {ccw }}$ to obtain the number of photons in the ccw mode.

\section{A. Rate equations for the electric field}

First a set of rate equations for the electric field inside the laser cavity is derived. As a starting point we take a traveling wave propagating in the $\mathrm{cw}$ direction, which can generally be described by

$$
\begin{aligned}
& \mathcal{E}_{\mathrm{cw}}(z, t)= \\
& \mathcal{E}_{\mathrm{cw}, 0} \exp \left(i(k z-\omega t)+\int_{0}^{z} \frac{\hat{g}\left(z^{\prime}\right)-\hat{\gamma}\left(z^{\prime}\right)}{2} d z^{\prime}\right) .
\end{aligned}
$$


Here $\mathcal{E}_{\mathrm{cw}, 0}$ is the amplitude of the field vector at $z=0, \omega$ is the optical frequency, $t$ is time, $k$ is the wave number, $z$ indicates the position along the cavity, $\hat{g}$ is the power gain per unit length and $\hat{\gamma}$ denotes the power loss per unit length. Since the points $z=0$ and $z=L$ represent the same point in the ring and the field in the cavity is assumed to be slowly varying compared to the round trip time (the slowly varying envelope approximation), it should hold that $\mathcal{E}_{\mathrm{cw}}(0, t)=\mathcal{E}_{\mathrm{cw}}(L, t)$, from which it is found that

$$
\mathcal{E}_{\mathrm{cw}}(z, t)=\exp \left(i k L+\frac{1}{2}(g-\gamma) L\right) \mathcal{E}_{\mathrm{cw}}(z, t),
$$

where $g L \equiv \int_{0}^{L} \hat{g}(z) d z$ is the round trip gain and $\gamma L \equiv$ $\int_{0}^{L} \hat{\gamma}(z) d z$ is the round trip loss.

$k$ is dependent on both the number of carriers, $n$, and optical frequency $\omega$. It is assumed that both $n$ and $\omega$ will be close to their threshold values when the laser is lasing, which justifies an expansion of $k$ around threshold. This yields

$$
k \approx k_{\mathrm{th}}+\left.\frac{\partial k}{\partial n}\right|_{\mathrm{th}}\left(n-n_{\mathrm{th}}\right)+\left.\frac{\partial k}{\partial \omega}\right|_{\mathrm{th}}\left(\omega-\omega_{\mathrm{th}}\right),
$$

where the subscript "th" denotes the value at threshold in the solitary laser. The effective refractive index is defined as $\mu_{e} \equiv k c / \omega$ such that $k=\mu_{e} \omega / c$. $\mu_{e}$ is dependent on both $n$ and $\omega$. This definition is substituted into (3) and the effective group index is defined as $\bar{\mu}_{e} \equiv \mu_{e}+\omega \partial \mu_{e} / \partial \omega$ to find

$$
k \approx \frac{\omega_{\mathrm{th}}}{c}\left(\mu_{e, \mathrm{th}}+\left.\frac{\partial \mu_{e}}{\partial n}\right|_{\mathrm{th}}\left(n-n_{\mathrm{th}}\right)+\frac{\bar{\mu}_{e}}{\omega_{\mathrm{th}}}\left(\omega-\omega_{\mathrm{th}}\right)\right),
$$

where $n_{\text {th }}$ is the number of carriers at threshold.

The frequency independent round trip gain $G_{1}$ and the frequency dependent round trip gain $G_{2}$ are now defined as

$$
\begin{aligned}
& G_{1} \equiv \\
& \exp \left(\frac{i \omega_{\mathrm{th}} L}{c}\left(\mu_{e, \mathrm{th}}+\frac{\partial \mu_{e}}{\partial n}\left(n-n_{\mathrm{th}}\right)\right)+\frac{1}{2}(g-\gamma) L\right) \\
& G_{2} \equiv \exp \left(i\left(\omega-\omega_{\mathrm{th}}\right) \tau_{r}\right)
\end{aligned}
$$

such that $\mathcal{E}_{\mathrm{cw}}(z, t)=G_{1} G_{2} \mathcal{E}_{\mathrm{cw}}(z, t)$. Where $\tau_{r} \equiv \bar{\mu}_{e} L / c$. Here it is assumed that the free spectral range of the filter is large compared to the linewidth of the laser, such that it can be considered spectrally flat. Since multiplication by $\exp \left(i \omega \tau_{r}\right)$ is equivalent to a time delay of $\tau_{r}$, the field at time $t$ can be expressed in terms of the electric field at time $t-\tau_{r}$ as

$$
\mathcal{E}_{\mathrm{cW}}(t)=G_{1} \exp \left(-i \omega_{\mathrm{th}} \tau_{r}\right) \mathcal{E}_{\mathrm{cw}}\left(t-\tau_{r}\right)
$$

It is now possible to define the slowly varying envelope $E_{\mathrm{cw}}(t)$ such that $\mathcal{E}_{\mathrm{cw}}(t)=\exp \left(-i \omega_{\text {th }} t\right) E_{\mathrm{cw}}(t)$. By substitution into (6) the envelope at time $t$ can be expressed in terms of the envelope at time $t-\tau_{r}$ as

$$
E_{\mathrm{cw}}(t)=G_{1} E_{\mathrm{cw}}\left(t-\tau_{r}\right)
$$

The round trip time is assumed to be short compared to the time scales with which the envelope $E_{\mathrm{cw}}(t)$ varies and the time derivative of $E_{\mathrm{cw}}(t)$ is approximated by a difference quotient such that the time derivative of the envelope becomes

$$
\begin{aligned}
\dot{E}_{\mathrm{cw}}(t) & \approx \frac{E_{\mathrm{cw}}(t)-E_{\mathrm{cw}}\left(t-\tau_{r}\right)}{\tau_{r}} \\
& \approx \frac{G_{1}-1}{\tau_{r}} E_{\mathrm{cw}}(t),
\end{aligned}
$$

where the dot in $\dot{E}_{\mathrm{cw}}(t)$ denotes a time derivative.

Subsequently the fraction in (8b) is linearized around threshold. To this end it is realized that $i \omega_{\mathrm{th}} \mu_{e, \mathrm{th}} L / c$ is an integer multiple of $2 \pi$ and does not contribute to $G_{1}$. When the laser is lasing, the gain compensates for almost all of the losses of the cavity. Therefore, the net gain, $g-\gamma \approx \partial g / \partial n\left(n-n_{\mathrm{th}}\right)$, is small while the laser is lasing such that the argument of the exponent in (5a) is small. Carrying out a linearization of $G_{1}$ around threshold and substituting the result into (8b) yields

$$
\dot{E}_{\mathrm{cw}}(t)=\frac{1}{2} \xi_{\mathrm{cw}}(1+i \alpha) N(t) E_{\mathrm{cw}}(t),
$$

where $N(t) \equiv n(t)-n_{\text {th }}$ and

$$
\xi_{\mathrm{cw}} \equiv \frac{L}{\tau_{r}} \frac{\partial g}{\partial n}, \quad \alpha \equiv \frac{2 \omega_{\mathrm{th}}}{c} \frac{\partial \mu_{e}}{\partial g} .
$$

A similar argument can be made for the electric field propagating in the ccw direction where the effect of the isolator is modeled by a gain difference by replacing $\gamma$ with $\gamma+\Delta \gamma$ in (5a). This yields

$$
\dot{E}_{\mathrm{ccw}}(t)=-\frac{1}{2} \Delta \Gamma E_{\mathrm{ccw}}(t)+\frac{1}{2} \xi_{\mathrm{ccw}}(1+i \alpha) N(t) E_{\mathrm{ccw}}(t),
$$

where

$$
\begin{gathered}
\xi_{\mathrm{ccw}} \equiv \exp \left(-\frac{1}{2} \Delta \gamma L\right) \xi_{\mathrm{cw}}, \\
\Delta \Gamma \equiv \frac{2-2 \exp \left(-\frac{1}{2} \Delta \gamma L\right)}{\tau_{r}} .
\end{gathered}
$$

$\Delta \Gamma$ models the gain difference that would be expected from the isolator. $\xi_{\mathrm{ccw}}$ is different from $\xi_{\mathrm{cw}}$, which is explained by the difference in threshold for both modes caused by the gain difference.

\section{B. Rate equations for the number of charge carriers}

To complete the deterministic model without EOF, a rate equation for the number of charge carriers is subsequently obtained. This equation is found by considering the SOA as a big reservoir of carriers where each carrier represents an electron in the excited state. Again a similar procedure was performed in [26] for a linear laser. The current analysis modifies this analysis slightly to include the effects of the two modes inherently present in a ring laser.

Several processes can be identified that add or remove carriers from this reservoir. First of all, the SOA is supplied with an injection current. This current adds carriers to the reservoir and is modeled by a rate $j(t)$.

Secondly, stimulated emission affects the number of carriers in addition to its effect on the number of photons in the cavity. Since each stimulated emission event consumes a carrier and produces a photon, the rate at which carriers are consumed is 
equal to the rate at which photons are generated. The latter can be found from (9) and (11). At steady state, stimulated emission compensates for the photons lost during a round trip, yielding a term $-\Gamma I$, where $I \equiv I_{\mathrm{cw}}+I_{\mathrm{ccw}}$ and $\Gamma \equiv(\exp (\gamma L)-1) / \tau_{r}$ represents all the optical losses in the entire cavity except the SOA, i.e. including the output coupling losses. Small deviations from steady state result in a slightly modified loss rate for the carriers. Combined, both effects result in a carrier loss rate of $-\left(\Gamma+\xi_{\mathrm{cw}} N(t)\right) I_{\mathrm{cw}}(t)-\left(\Gamma+\xi_{\mathrm{ccw}} N(t)\right) I_{\mathrm{ccw}}(t)$.

All other processes that have an effect on the number of carriers in the SOA are grouped together. Their effect on the number of carriers is linearized around threshold with respect to the number of carriers. This yields an average carrier lifetime, $T$, and a resulting loss of carriers $n(t) / T$. Together, these terms yield

$$
\begin{aligned}
\dot{n}(t)= & j(t)-\frac{n(t)}{T}-\left(\Gamma+\xi_{\mathrm{cw}} N(t)\right) I_{\mathrm{cw}}(t) \\
& -\left(\Gamma+\xi_{\mathrm{ccw}} N(t)\right) I_{\mathrm{ccw}}(t) .
\end{aligned}
$$

By substituting $n(t)=n_{\mathrm{th}}+N(t)$ and $J(t)=j(t)-n_{\mathrm{th}} / T$, one obtains

$$
\begin{aligned}
\dot{N}(t)= & J(t)-\frac{N(t)}{T}-\left(\Gamma+\xi_{\mathrm{cw}} N(t)\right) I_{\mathrm{cw}}(t) \\
& -\left(\Gamma+\xi_{\mathrm{ccw}} N(t)\right) I_{\mathrm{ccw}}(t) .
\end{aligned}
$$

\section{External optical feedback and noise}

To the deterministic model, effects of EOF are added according to [30], where it is realized that light only couples from the $\mathrm{cw}$ into the ccw mode and not the other way around. This is only true if the effect of backscattering can be neglected. For weakly guiding waveguides, such as those used in modern InP platforms, this is generally true as the backscattering is approximately $-50 \mathrm{~dB} \mathrm{~mm}^{-1}$ [31]. The intensity of the slowly varying envelope, $I_{x}(t)$, and the phase, $\phi_{x}(t)$, are defined such that $E_{x}(t)=\sqrt{I_{x}(t)} \exp \left(i \phi_{x}(t)\right)$ where $x$ represents the cw or ccw mode. By separating the real and imaginary parts of the rate equations a new set of equations is found for the intensity and phase of the light in both modes. Together with the rate equation for the carriers, this results in a set of five equations. Noise terms are added to these equations as was done in [32]. On average, intensity noise will contribute photons at a rate $R_{s}$ to each mode. The average contribution to the phase and the number of carriers equals 0 . We define $F_{y}$ to be the zeroaverage noise, where $y$ indicates the quantity to which the noise is added. Finally, this results in the equations

$$
\begin{aligned}
\dot{I}_{\mathrm{cw}}(t)= & \xi_{\mathrm{cw}} N(t) I_{\mathrm{cw}}(t)+R_{s}+F_{I, \mathrm{cw}}(t) \\
\dot{I}_{\mathrm{ccw}}(t)= & \left(\xi_{\mathrm{ccw}} N(t)-\Delta \Gamma\right) I_{\mathrm{ccw}}(t)+R_{s}+F_{I, \mathrm{ccw}}(t) \\
& +|\kappa| \sqrt{I_{\mathrm{cw}}(t-\tau) I_{\mathrm{ccw}}(t)} \cos \left(\psi_{0}+\Delta \phi_{\tau}(t)\right) \\
\dot{\phi}_{\mathrm{cw}}(t)= & \frac{1}{2} \xi_{\mathrm{cw}} \alpha N(t)+F_{\phi, \mathrm{cw}}(t) \\
\dot{\phi}_{\mathrm{ccw}}(t)= & \frac{1}{2} \xi_{\mathrm{ccw}} \alpha N(t)+F_{\phi, \mathrm{ccw}}(t) \\
& +\frac{1}{2}|\kappa| \sqrt{\frac{I_{\mathrm{cw}}(t-\tau)}{I_{\mathrm{ccw}}(t)}} \sin \left(\psi_{0}+\Delta \phi_{\tau}(t)\right)
\end{aligned}
$$

$$
\begin{aligned}
\dot{N}(t)= & J-\frac{N(t)}{T}-\left(\Gamma+\xi_{\mathrm{cw}} N(t)\right) I_{\mathrm{cw}}(t) \\
& -\left(\Gamma+\xi_{\mathrm{ccw}} N(t)\right) I_{\mathrm{ccw}}(t)+F_{N}(t) .
\end{aligned}
$$

Here $R_{s}$ is the rate of spontaneous emission into a single mode, $|\kappa| \equiv \sqrt{T_{\text {iso }} T_{\text {out }}^{2} R} / \tau_{r}$ is the feedback rate, $T_{\text {iso }}$ is defined as the power transmission of the isolator for the $\mathrm{ccw}$ mode relative to the transmission for the $\mathrm{cw}$ mode and represents the isolation provided by the isolator, $T_{\text {out }}$ is the power transmission coupled out of the cavity by the output coupler, $R$ is the power reflection of the external reflector, $\arg \kappa$ is the change in phase the light accumulates while propagating outside the laser cavity, $\tau$ is the feedback delay time, $\Delta \phi_{\tau}(t) \equiv \phi_{\mathrm{cw}}(t-\tau)-\phi_{\mathrm{ccw}}(t)$ is the phase difference between the field in the cw and ccw modes and $\psi_{0} \equiv \arg \kappa-\omega_{\text {th }} \tau$ is the phase delay due to propagation outside of the laser cavity.

\section{Steady state values}

Using the model, the steady state values are obtained. It is assumed that the intensity and frequency of the light eventually reach a steady state, represented by a fixed point in the three dimensional state space spanned by $I_{\mathrm{cw}}, I_{\mathrm{ccw}}$ and $N$. Because of the noise terms, $F_{y}$, the laser will make minor excursions around this point. Assuming these excursions are small compared to any non-linearities in the laser, it is possible to find the steady state values from (15) by neglecting these small excursions, i.e. by taking $F_{y}(t)=0$. It follows from (15a) that

$$
\bar{N}=-\frac{R_{s}}{\xi_{\mathrm{cw}} \bar{I}_{\mathrm{cw}}}
$$

where the bar denotes that the value is only valid in the steady state.

From (15c) and (15d) it follows that $\Delta \phi_{\tau}(t)$ becomes time independent in the steady state. Assuming $|\kappa| \sqrt{\bar{I}_{\mathrm{cw}} / \bar{I}_{\mathrm{ccw}}} \gg$ $\left(\xi_{\mathrm{cw}}-\xi_{\mathrm{ccw}}\right) \alpha \bar{N}$, the time-independent solution of the resulting differential equation yields $\sin \left(\psi_{0}+\Delta \phi_{\tau}\right) \approx 0$ and $\cos \left(\psi_{0}+\right.$ $\left.\Delta \phi_{\tau}\right) \approx 1$. The latter result can be used to simplify (15b), removing its dependency on the feedback phase. This can be explained because the EOF is the dominant effect coupling the two modes directly. This allows the phase of the ccw mode to lock to the phase of the ccw mode. In other words, a change in feedback phase is accompanied by a change in the phase difference between the two modes. The resulting simplified, quadratic equation can then be solved for $\bar{I}_{\text {ccw }}$ yielding

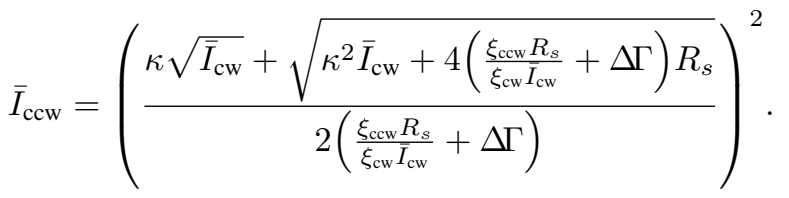

where it is worthwhile to notice that $\bar{I}_{\mathrm{ccw}}=\bar{I}_{\mathrm{cw}} \kappa^{2} / \Delta \Gamma^{2}$ for $R_{s} \downarrow 0$ as it provides an approximation for the ratio of optical power in both modes.

It is possible to express (15e) in terms of $\bar{I}_{\mathrm{cw}}$ using these results, and it is found that

$$
\begin{aligned}
0= & J-\Gamma\left(\bar{I}_{\mathrm{cw}}+\bar{I}_{\mathrm{ccw}}\left(\bar{I}_{\mathrm{cw}}\right)\right) \\
& -N\left(\bar{I}_{\mathrm{cw}}\right)\left(\frac{1}{T}+\xi_{\mathrm{cw}} \bar{I}_{\mathrm{cw}}+\xi_{\mathrm{ccw}} \bar{I}_{\mathrm{ccw}}\left(\bar{I}_{\mathrm{cw}}\right)\right)
\end{aligned}
$$


Table I

VALUES USED FOR PHYSICAL QUANTITIES

\begin{tabular}{llll}
\hline Description & Parameter & value & unit \\
\hline Differential gain & $\xi$ & $10^{3}$ & $\mathrm{~s}^{-1}$ \\
Injection current over threshold & $J$ & $10^{17}$ & $\mathrm{~s}^{-1}$ \\
Loss rate & $\Gamma$ & $10^{11}$ & $\mathrm{~s}^{-1}$ \\
Carrier life time & $T$ & $10^{-9}$ & $\mathrm{~s}^{-1}$ \\
Spontaneous emission rate & $R_{s}$ & $10^{11}$ & $\mathrm{~s}^{-1}$ \\
Feedback delay time & $\tau$ & $10^{-7}$ & $\mathrm{~s}$ \\
Cavity length & $L$ & 2 & $\mathrm{~cm}$ \\
Relative transmission of isolator & $T_{\text {iso }}$ & 3 & $\mathrm{~dB}$ \\
Outcoupler transmission & $T_{\text {out }}$ & 3 & $\mathrm{~dB}$ \\
External reflectivity & $R$ & -20 & $\mathrm{~dB}$ \\
Group index & $\mu_{e}$ & 3.7 & \\
Linewidth enhancement parameter & $\alpha$ & 3 & \\
\hline
\end{tabular}

Numerically solving this equation yields a value for $\bar{I}_{\mathrm{cw}}$ which can subsequently be used to find $\bar{I}_{\text {ccw }}$ and $\bar{N}$.

The output power is studied numerically, using the parameter values indicated in table I. Most of these values are typical for semiconductor lasers. To give a feeling for the magnitude of these numbers, the following equalities might prove insightful The injection rate, $J$, is equivalent to an injection current of approximately $16 \mathrm{~mA}$ over the threshold current. The photon lifetime, $1 / \Gamma$, is equivalent to a loss of $6.5 \mathrm{~dB} \mathrm{~cm}^{-1}$ The feedback delay time, $\tau$, is equivalent to a reflection at approximately $10 \mathrm{~m}$ from the laser. To improve process tolerances, coupling of the ring cavity to the output waveguide is done using multimode interferometers. These components usually act as $3 \mathrm{~dB}$-splitters, which explains the number for $T_{\text {out }}$.

The cavity length, $L$, is taken rather long, and is usually on the order of $100 \mu \mathrm{m}$. Inclusion of an isolator such as presented in [25] does however require the addition of several components to the laser cavity. These are two phase modulators and a spectral filter. The phase modulators each need to sinusoidally modulate the optical phase with an amplitude of approximately $1.2 \mathrm{rad}$ at a frequency of several $\mathrm{GHz}$ requiring a length of several $\mathrm{mm}$ each. The spectral filter requires a free spectral range of several $\mathrm{GHz}$ and can be implemented using serial Mach-Zehnder filters. This requires about $1 \mathrm{~cm}$ of waveguides. Finally the SOA needs to supply more gain to compensate for the extra losses that are introduced by the phase modulators and the spectral filter increasing its length. $2 \mathrm{~cm}$ is therefore taken as a more appropriate cavity length.

The simulation results for $I_{\mathrm{cw}}$ are found in Fig. 2. This figure shows trends that are expected. $I_{\mathrm{cw}}$ decreases for increasing amounts of EOF, while an increase in isolation counteracts this change. As EOF increases, $I_{\text {ccw }}$ will start to grow, consuming a number of carriers while doing so. Since both the cw and $\mathrm{ccw}$ modes interact with the same carrier population, this results in a reduction of $I_{\mathrm{cw}}$. When the laser receives strong EOF compared to the gain difference inside the cavity, the $\mathrm{ccw}$ mode will become the lasing mode. In this regime the cw mode provides very little output power and it is therefore not interesting to operate the laser in this regime. If the amount of isolation is adequate, this effect becomes negligible however.

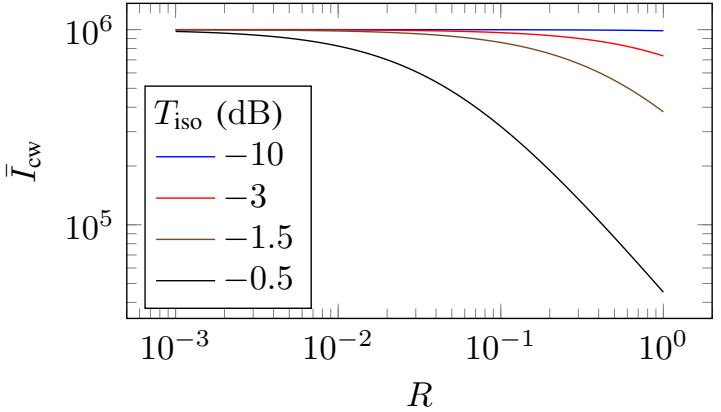

Figure 2. The steady state output power as a function of feedback strength as indicated in figure 1. For stronger EOF, the ccw mode experiences a higher effective gain and is therefore stronger. Since the carriers are shared between both modes, this results in a slightly weaker cw mode. It can also be seen that for stronger isolation, i.e. small $T_{\text {iso }}$, this effect is reduced. When $100 \%$ of the light is returned to the cavity as EOF, the effect on $I_{\mathrm{cw}}$ is limited to $2 \%$ for an isolation of $10 \mathrm{~dB}$

\section{SMALl SIGNAL ANALYSIS}

To predict the dynamic behavior of the laser, the changes in field and carriers are linearized around steady state. Assuming the fluctuations in field strength and carrier number are small compared to their steady state values, the laser is best modeled by a system of linear equations that is driven by the noise forces. The problem is subsequently converted to the frequency domain by making use of a Fourier transform, $2 \pi x(t)=\int \exp (i \omega t) x(\omega) \mathrm{d} \omega$. Finally it is realized that the deterministic phases of both modes lock to each other owing to (15d), meaning that $\dot{I}_{\mathrm{cw}}, \dot{I}_{\mathrm{ccw}}$ and $\dot{N}$ are all independent of $\dot{\phi}_{\mathrm{cw}}$ and $\dot{\phi}_{\mathrm{ccw}}$. This allows for a study of the behavior of the intensities and carrier number independent of the phases and results in

$$
\left(\begin{array}{c}
F_{I, \mathrm{cw}}(\omega) \\
F_{I, \mathrm{ccw}}(\omega) \\
F_{N}(\omega)
\end{array}\right)=Z\left(\begin{array}{c}
\mathrm{d} I_{\mathrm{cw}}(\omega) \\
\mathrm{d} I_{\mathrm{ccw}}(\omega) \\
\mathrm{d} N(\omega)
\end{array}\right)
$$

where

$$
Z=\left(\begin{array}{ccc}
i \omega-\xi_{\mathrm{cw}} \bar{N} & 0 & -\xi_{\mathrm{cw}} \bar{I}_{\mathrm{cw}} \\
\zeta_{\mathrm{ccw}, \mathrm{cw}} & \zeta_{\mathrm{ccw}, \mathrm{ccw}} & -\xi_{\mathrm{ccw}} \bar{I}_{\mathrm{ccw}} \\
\Gamma+\xi_{\mathrm{cw}} \bar{N} & \Gamma+\xi_{\mathrm{ccw}} \bar{N} & \zeta_{\mathrm{N}, \mathrm{N}}
\end{array}\right),
$$

$\zeta_{\mathrm{ccw}, \mathrm{cw}}=-\frac{1}{2} \frac{|\kappa|^{2}}{\Delta \Gamma} \exp (-i \omega \tau), \zeta_{\mathrm{ccw}, \mathrm{ccw}}=i \omega-\xi_{\mathrm{ccw}} \bar{N}+\frac{1}{2} \Delta \Gamma$ and $\zeta_{\mathrm{N}, \mathrm{N}}=i \omega+\frac{1}{T}+\xi_{\mathrm{cw}} \bar{I}_{\mathrm{cw}}+\xi_{\mathrm{ccw}} \bar{I}_{\mathrm{ccw}}$. Let the first vector be called $\mathbf{F}$, the matrix $\mathbf{Z}$ and the last vector $d \mathbf{X}$. $\mathbf{Z}$ is the linearized coupling between the various deviations from steady state and is found from (15). It is now possible to express $d \mathbf{X}$ in terms of $\mathbf{F}$ and $\mathbf{Z}$ by matrix inversion, e.g. through Cramers'

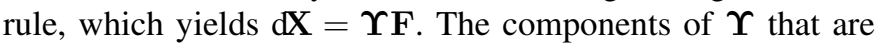
used in this paper can be found in the Appendix.

\section{A. Relative Intensity Noise}

From $d \mathbf{X}$ it is possible to find the RIN spectrum which is defined as

$$
\operatorname{RIN}(\omega) \equiv \frac{\left|\mathrm{d} I_{\mathrm{cw}}(\omega)\right|^{2}}{\bar{I}_{\mathrm{cw}}^{2}}
$$

and where

$$
\begin{aligned}
\mathrm{d} I_{\mathrm{cw}}(\omega)= & v_{\mathrm{cw}, \mathrm{cw}}(\omega) F_{I, \mathrm{cw}}(\omega)+v_{\mathrm{cw}, \mathrm{ccw}}(\omega) F_{I, \mathrm{ccw}}(\omega) \\
& +v_{\mathrm{cw}, N}(\omega) F_{N}(\omega)
\end{aligned}
$$


following from the matrix inversion. It is apparent that terms of the form $F_{I, \text { cw }}^{*}(\omega) F_{I, \text { ccw }}(\omega)$ will appear when calculating the RIN. These are found using the procedure outlined in [32], and result in

$$
\begin{aligned}
\left\langle\left|F_{I, \mathrm{cw}}(\omega)\right|^{2}\right\rangle & =R_{s}\left(2 \bar{I}_{\mathrm{cw}}+1\right) \\
\left\langle\left|F_{I, \mathrm{ccw}}(\omega)\right|^{2}\right\rangle & =R_{s}\left(2 \bar{I}_{\mathrm{ccw}}+1\right) \\
\left\langle\left|F_{\phi, \mathrm{cw}}(\omega)\right|^{2}\right\rangle & =\frac{R_{s}}{2 \bar{I}_{\mathrm{cw}}} \\
\left\langle\left|F_{\phi, \mathrm{ccw}}(\omega)\right|^{2}\right\rangle & =\frac{R_{s}}{2 \bar{I}_{\mathrm{ccw}}} \\
\left\langle\left|F_{N}(\omega)\right|^{2}\right\rangle & =2 R_{s} \\
\left\langle F_{I, \mathrm{cw}}(\omega)^{*} F_{N}(\omega)\right\rangle & =-R_{s} \\
\left\langle F_{I, \mathrm{ccw}}(\omega)^{*} F_{N}(\omega)\right\rangle & =-R_{s} .
\end{aligned}
$$

Note that $R_{s}$ is the rate of spontaneous emission into one mode, and consequently a factor 2 is found in (23e).

The components of $\Upsilon$ together with (23) now provide a means for calculating the RIN spectrum. The equations for the gain difference $\Delta \Gamma$ and feedback rate $\kappa$ are expressed in terms of the reflectivity of the external reflection causing the feedback, $R$, and the relative transmission of the isolator, $T_{\text {iso. }}$. It is realized that this number can be related to the gain difference induced by the isolator as $T_{\text {iso }}=\exp (-\Delta \gamma L)$ and it is found from the definition of $\Delta \Gamma$ that $\Delta \Gamma=2\left(1-\sqrt{T_{\text {iso }}}\right) / \tau_{r}$. The feedback rate signifies the number of photons that return to the laser cavity each second, relative to the number of photons present in the cavity. During each round trip of the light, a fraction $T_{\text {out }}$ will couple out of the cavity. A fraction $R$ of this light will be reflected back towards the laser and finally a fraction $T_{\text {out }}$ of the reflected light will couple back into the cavity. In the cavity the light first passes through the isolator before it enters the SOA. Since most of the interactions between the modes and carriers occur in the SOA, only the fraction of photons that passes the isolator is considered as EOF. It then follows that $\kappa=\sqrt{T_{\text {iso }} T_{\text {out }}^{2} R} / \tau_{r}$.

The previous results are studied numerically to find effects of EOF on the RIN and linewidth of the laser. To this end (22) is substituted into (21) which can then be solved using $\bar{I}_{\mathrm{cw}}$, $\bar{I}_{\text {ccw }}$ and $\bar{N}$ obtained from the steady state analysis. As with the steady state analysis, the parameter values indicated in table I were used unless indicated otherwise.

The RIN spectrum is found as shown in Fig. 3. From this figure it is apparent that the amount of EOF only significantly influences the RIN at low frequencies. To highlight this dependency, Fig. 4 shows the RIN in the limit for $\omega \downarrow 0$ as a function of $R$ and $T_{\text {iso. This figure shows that the low }}$ frequency RIN is fairly constant for small values of $R$. After some maximum feedback strength, the low frequency RIN starts to increase rapidly however. This point is dependent on $T_{\text {iso }}$ and from this we can derive the maximum amount of EOF that is adequately suppressed by the isolator. For an isolation of $10 \mathrm{~dB}$ and feedback of $-0.1 \mathrm{~dB}$, i.e. almost all of the laser light is reflected back into the cavity, the RIN only increases by a factor of 2 . When compared to the $60 \mathrm{~dB}$ isolation traditionally required to fully suppress the effects of $\mathrm{EOF}$, this is a substantial improvement.

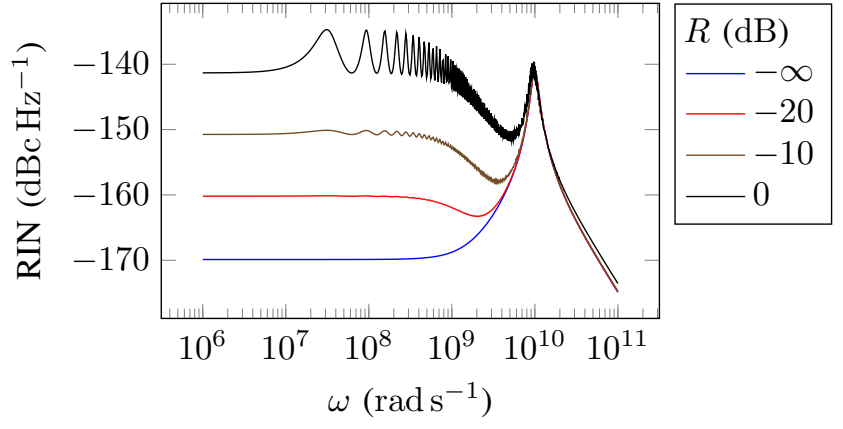

Figure 3. RIN spectrum for various values of $R$, using the values specified in table I for the intracavity isolation and other parameters. The relaxation oscillation frequency is clearly visible as the peak. Especially for higher amounts of feedback, there is a clear ripple in the spectrum. This ripple is caused by a resonance via the external reflection, the ccw mode and the carriers. The frequencies at which these ripples occure are directly related to the feedback delay time $\tau$. For lower and higher frequencies the RIN changes in a similar way as for the frequencies shown. Increasing amounts of EOF mainly result in an increase in the RIN at low frequencies.

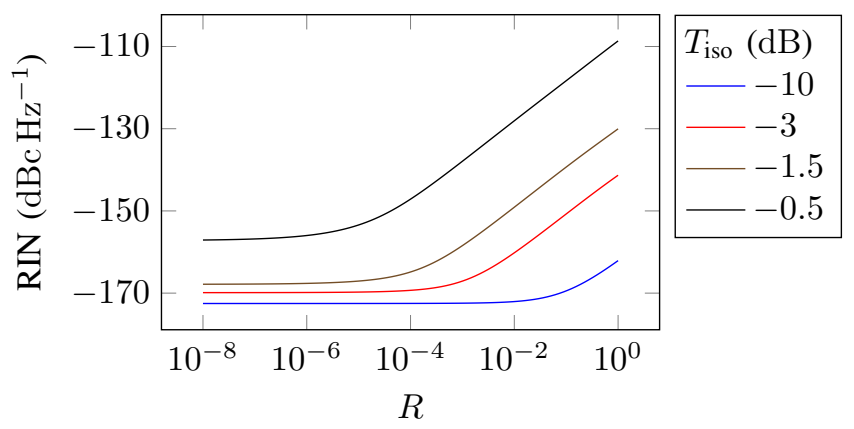

Figure 4. Low frequency RIN as a function of $R$ and $T_{\text {iso. The red curve }}$ corresponds to the values in Fig. 3 for $\omega \downarrow 0$. It is clear that higher amounts of isolation provide a higher immunity to EOF.

\section{B. Linewidth}

The linewidth of the laser is obtained by considering the deviations from the steady state in (15c). It is found that

$$
\mathrm{d} \Delta \nu(\omega)=\frac{1}{2 \pi}\left(\frac{1}{2} \xi_{\mathrm{cw}} \alpha \mathrm{d} N(\omega)+F_{\phi, \mathrm{cw}}(\omega)\right) .
$$

The first term of this equation can be expanded using $\mathrm{d} \mathbf{X}=\Upsilon \mathbf{Y F}$. The power spectral density of the instantaneous frequency deviation is then equal to $|\mathrm{d} \Delta \nu(\omega)|^{2}$ and the linewidth is its limit for $\omega \rightarrow 0$. The noise correlations in the resulting equation are found according to equations 23 . The power spectral density of the frequency noise is then found as in Fig. 5.

The linewidth is equal to the frequency noise for $\omega \downarrow 0$. Fig. 6 shows its value as a function of both $R$ and $T_{\text {iso. }}$. It is clear from this picture that even for very small isolation, the changes in linewidth remain small. Furthermore, the change in linewidth can almost completely be attributed to the reduction of the power in the mode, $\bar{I}_{\mathrm{cw}}$, due to cross-gain saturation. It follows approximately a $1 / \bar{I}_{\mathrm{cw}}$ trend, similar to what would be expected for a Fabry-Pérot laser. Even for $R=1,3 \mathrm{~dB}$ of isolation is sufficient to reduce the change in linewidth to approximately $18 \% .10 \mathrm{~dB}$ of isolation even reduces this number to $1 \%$. As with the RIN this is in stark contrast to the $60 \mathrm{~dB}$ that is traditionally required. 


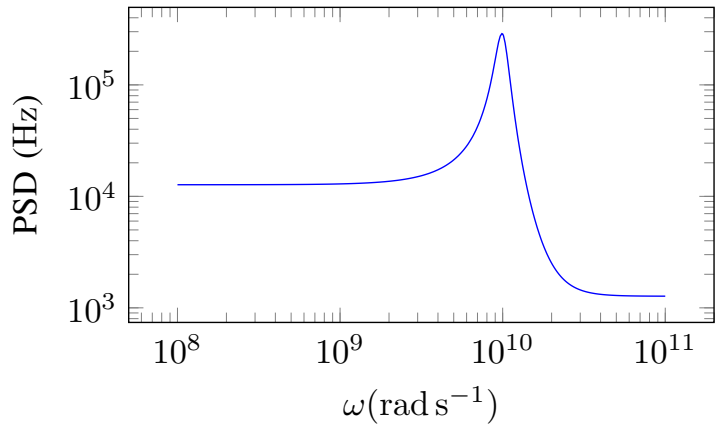

Figure 5. Power spectral density of the frequency deviations. The relaxation oscillation frequency is clearly recognizable as the peak. The linewidth is obtained from the limit to low frequency.

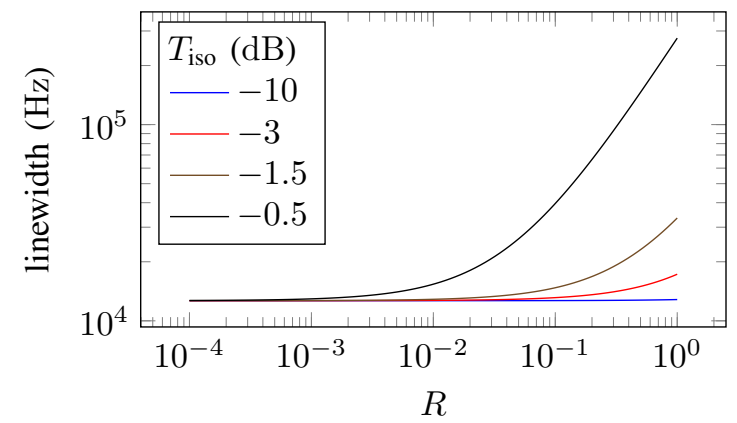

Figure 6. Linewidth of the laser as function of the amount of feedback and isolation. It is clear that the linewidth increases for increasing EOF. This figure also shows that this effect is mitigated by an increase in intracavity isolation.

\section{CONCLUSION}

In this paper a novel type of integrated laser was theoretically analysed. The cavity of this laser includes a weak optical isolator to provide a gain difference between the two propagation directions. This forces the laser to lase unidirectionally. Because the EOF returns to another, sub-threshold mode, external cavity modes are non-existent in this type of laser and the influence of EOF on the laser output is very small.

Since the required isolation is less than $10 \mathrm{~dB}$, the isolator can be implemented on-chip. The output coupler can be implemented using a multi-mode interferometer and the spectral filter can be fabricated using a series of asymmetric MachZehnder interferometers as was done in [33]. As all components of the laser have already been demonstrated, this type of laser is a viable candidate for realization.

The RIN spectrum and linewidth were calculated as indicators of the stability of the laser output. Both of these properties were obtained as a function of both the feedback rate and the intracavity isolation by utilizing a rate equation analysis. From this analysis it follows that only $3 \mathrm{~dB}$ of intracavity isolation is sufficient to make the laser immune to $-30 \mathrm{~dB}$ of EOF. For $10 \mathrm{~dB}$ of isolation it is found that EOF as strong as $-0.1 \mathrm{~dB}$ does not affect the laser to a great extend. In that case the changes in RIN are limited to a factor 2 and are calculated to be $6.4 \times 10^{-17} \mathrm{rad}^{-1}$. The intensity of the lasing mode and its linewidth are affected by approximately $1 \%$.

The main advantage of integrating the isolator inside the laser cavity as opposed to outside the cavity is the greatly reduced amount of isolation required, making it much easier to realize. Such a weak isolator also shows less insertion loss. This can potentially be leveraged to increase the wall plug efficiency of the laser, while maintaining feedback insensitivity.

\section{APPENDIX}

The small signal coefficients used in this paper can be found by inverting matrix $\mathbf{Z}$ from (19). They yield

$$
\begin{aligned}
\mathbf{Z}^{-1} & =\Upsilon \\
& =\left(\begin{array}{lll}
v_{\mathrm{cw}, \mathrm{cw}}(\omega) & v_{\mathrm{cw}, \mathrm{ccw}}(\omega) & v_{\mathrm{cw}, N}(\omega) \\
v_{\mathrm{ccw}, \mathrm{cw}}(\omega) & v_{\mathrm{ccw}, \mathrm{ccw}}(\omega) & v_{\mathrm{ccw}, N}(\omega) \\
v_{N, \mathrm{cw}}(\omega) & v_{N, \mathrm{ccw}}(\omega) & v_{N, N}(\omega)
\end{array}\right),
\end{aligned}
$$

where

$$
\begin{aligned}
& v_{\mathrm{cw}, \mathrm{cw}}(\omega)= \frac{1}{\Delta}\left(\bar{I}_{\mathrm{ccw}} \xi_{\mathrm{ccw}}\left(\Gamma+\xi_{\mathrm{ccw}} \bar{N}\right)\right. \\
&+\left(i \omega-\xi_{\mathrm{ccw}} \bar{N}+\frac{\Delta \Gamma}{2}\right) \\
&\left.\left(i \omega+\frac{1}{T}+\xi_{\mathrm{cw}} \bar{I}_{\mathrm{cw}}+\xi_{\mathrm{ccw}} \bar{I}_{\mathrm{ccw}}\right)\right) \\
& v_{\mathrm{cw}, \mathrm{ccw}}(\omega)=-\frac{1}{\Delta}\left(\bar{I}_{\mathrm{cw}} \xi_{\mathrm{cw}}\left(\Gamma+\xi_{\mathrm{ccw}} \bar{N}\right)\right) \\
& v_{\mathrm{cw}, N}(\omega)= \frac{1}{\Delta}\left(\bar{I}_{\mathrm{cw}} \xi_{\mathrm{cw}}\left(i \omega-\xi_{\mathrm{ccw}} \bar{N}+\frac{\Delta \Gamma}{2}\right)\right) \\
& v_{N, \mathrm{cw}}(\omega)=-\frac{1}{\Delta}\left(\Gamma \left(i \omega-\xi_{\mathrm{ccw}} \bar{N}+\frac{\Delta \Gamma}{2}\right.\right. \\
&\left.+\frac{|\kappa|^{2}}{2 \Delta \Gamma} \exp (-i \omega \tau)\right) \\
& \quad+\frac{\xi_{\mathrm{ccw}}|\kappa|^{2}}{2 \Delta \Gamma} \exp (-i \omega \tau) \\
&\left.\left.+\xi_{\mathrm{cw}}\left(i \omega-\xi_{\mathrm{ccw}} \bar{N}+\frac{\Delta \Gamma}{2}\right)\right)\right) \\
& v_{N, \mathrm{ccw}}(\omega)= \frac{1}{\Delta}\left(i \omega-\xi_{\mathrm{cw}} \bar{N}\right)\left(\Gamma+\xi_{\mathrm{ccw}} \bar{N}\right) \\
& v_{N, N}(\omega)= \frac{1}{\Delta}\left(\frac{1}{2} i \omega \Delta \Gamma-\omega_{\mathrm{ccw}} \bar{N}^{2}-i \omega \bar{N}\left(\xi_{\mathrm{cw}}+\xi_{\mathrm{ccw}}\right)+\right. \\
&\left.\frac{1}{2} \xi_{\mathrm{cw}} \bar{N} \Delta \Gamma\right)
\end{aligned}
$$

are the components of this matrix that are used in this paper to calculate the RIN and linewidth of the cw mode. $\Delta$ is the determinant of $\mathbf{Z}$.

\section{REFERENCES}

[1] R. Tkach and A. Chraplyvy, "Regimes of feedback effects in $1.5-\mu \mathrm{m}$ distributed feedback lasers," Journal of Lightwave Technology, vol. 4, no. 11, pp. 1655-1661, Nov 1986.

[2] O. Hirota and Y. Suematsu, "Noise properties of injection lasers due to reflected waves," IEEE Journal of Quantum Electronics, vol. 15, no. 3 , pp. 142-149, Mar 1979.

[3] K. Stubkjaer and M. Small, "Noise properties of semiconductor lasers due to optical feedback," IEEE Journal of Quantum Electronics, vol. 20 , no. 5, pp. 472-478, May 1984. 
[4] M. F. Alam, M. A. Karim, and S. Islam, "Effects of structural parameters on the external optical feedback sensitivity in DFB semiconductor lasers," IEEE Journal of Quantum Electronics, vol. 33, no. 3, pp. 424-433, Mar 1997.

[5] J. Ballato and E. Snitzer, "Fabrication of fibers with high rare-earth concentrations for Faraday isolator applications," Appl. Opt., vol. 34 , no. 30, pp. 6848-6854, Oct 1995.

[6] A. E. Turner, R. L. Gunshor, and S. Datta, "New class of materials for optical isolators," Appl. Opt., vol. 22, no. 20, pp. 3152-3154, Oct 1983

[7] L. Sun, S. Jiang, J. D. Zuegel, and J. R. Marciante, "All-fiber optical isolator based on Faraday rotation in highly terbium-doped fiber," Opt Lett., vol. 35, no. 5, pp. 706-708, Mar 2010.

[8] T. R. Zaman, X. Guo, and R. J. Ram, "Semiconductor waveguide isolators," Journal of Lightwave Technology, vol. 26, no. 2, pp. 291-301, Jan 2008.

[9] R. L. Espinola, T. Izuhara, M.-C. Tsai, R. M. Osgood, and H. Dötsch, "Magneto-optical nonreciprocal phase shift in garnet/silicon-on-insulator waveguides," Opt. Lett., vol. 29, no. 9, pp. 941-943, May 2004.

[10] L. Bi, J. Hu, P. Jiang, D. H. Kim, G. F. Dionne, L. C. Kimerling, and C. A. Ross, "On-chip optical isolation in monolithically integrated nonreciprocal optical resonators," Nat Photon, vol. 5, no. 12, pp. 758-762, Dec 2011.

[11] S. Yamamoto, Y. Okamura, and T. Makimoto, "Analysis and design of semileaky-type thin-film optical waveguide isolator," IEEE Journal of Quantum Electronics, vol. 12, no. 12, pp. 764-770, December 1976.

[12] K. Ando, T. Okoshi, and N. Koshizuka, "Waveguide magneto-optic isolator fabricated by laser annealing," Applied Physics Letters, vol. 53 , no. 1 , pp. 4-6, 1988 .

[13] M. Levy, R. M. Osgood, H. Hegde, F. J. Cadieu, R. Wolfe, and V. J Fratello, "Integrated optical isolators with sputter-deposited thin-film magnets," IEEE Photonics Technology Letters, vol. 8, no. 7, pp. 903-905, July 1996

[14] T. Shintaku, "Integrated optical isolator based on efficient nonreciprocal radiation mode conversion," Applied Physics Letters, vol. 73, no. 14, pp. 1946-1948, 1998.

[15] H. Dötsch, N. Bahlmann, O. Zhuromskyy, M. Hammer, L. Wilkens, R. Gerhardt, P. Hertel, and A. F. Popkov, "Applications of magnetooptical waveguides in integrated optics: review," J. Opt. Soc. Am. B, vol. 22 , no. 1 , pp. 240-253, Jan 2005.

[16] J. Fujita, M. Levy, R. M. Osgood Jr., L. Wilkens, and H. Dötsch, "Waveguide optical isolator based on Mach-Zehnder interferometer," Applied Physics Letters, vol. 76, no. 16, pp. 2158-2160, 2000.

[17] Y. Shoji, T. Mizumoto, H. Yokoi, I.-W. Hsieh, and R. M. Osgood Jr., "Magneto-optical isolator with silicon waveguides fabricated by direct bonding," Applied Physics Letters, vol. 92, no. 7, p. 071117, 2008.

[18] M.-C. Tien, T. Mizumoto, P. Pintus, H. Kromer, and J. E. Bowers, "Silicon ring isolators with bonded nonreciprocal magneto-optic garnets," Opt. Express, vol. 19, no. 12, pp. 11740-11 745, Jun 2011.

[19] Y. Shoji and T. Mizumoto, "Magneto-optical non-reciprocal devices in silicon photonics," Science and Technology of Advanced Materials, vol. 15, no. 1, p. 014602, 2014.

[20] L. Fan, J. Wang, L. T. Varghese, H. Shen, B. Niu, Y. Xuan, A. M. Weiner, and M. Qi, "An all-silicon passive optical diode," Science, vol. 335, no. 6067, pp. 447-450, 2012.

[21] M. J. R. Heck, S. Srinivasan, M. L. Davenport, and J. E. Bowers, "Integrated microwave photonic isolators: Theory, experimental realization and application in a unidirectional ring mode-locked laser diode," Photonics, vol. 2, no. 3, pp. 957-968, 2015.

[22] S. Bhandare, S. K. Ibrahim, D. Sandel, H. Zhang, F. Wust, and R. Noe, "Novel nonmagnetic 30-dB traveling-wave single-sideband optical isolator integrated in III/V material," IEEE Journal of Selected Topics in Quantum Electronics, vol. 11, no. 2, pp. 417-421, March 2005.

[23] H. Lira, Z. Yu, S. Fan, and M. Lipson, "Electrically driven nonreciprocity induced by interband photonic transition on a silicon chip," Phys. Rev Lett., vol. 109, p. 033901, Jul 2012.

[24] P. Dong, "Travelling-wave Mach-Zehnder modulators functioning as optical isolators," Opt. Express, vol. 23, no. 8, pp. 10498-10 505, Apr 2015.

[25] C. R. Doerr, N. Dupuis, and L. Zhang, "Optical isolator using two tandem phase modulators," Opt. Lett., vol. 36, no. 21, pp. 4293-4295, Nov 2011.

[26] K. Petermann, Laser diode modulation and noise. Kluwer Academic Publishers, 1988.

[27] G. Morthier and P. Mechet, "Theoretical analysis of unidirectional operation and reflection sensitivity of semiconductor ring or disk lasers," IEEE Journal of Quantum Electronics, vol. 49, no. 12, pp. 1097-1101, Dec 2013
[28] T. T. M. van Schaijk, E. A. J. M. Bente, and D. Lenstra, "Design of feedback insensitive InP ring laser," in Proceedings of the 20th Annual Symposium of the IEEE Photonics Benelux Chapter, 2015.

[29] P. van Schaijk, D. Lenstra, and E. Bente, "Feedback-insensitive integrated laser," in Proceedings of the 18th European Conference on Integrated Optics, 2016.

[30] R. Lang and K. Kobayashi, "External optical feedback effects on semiconductor injection laser properties," IEEE Journal of Quantum Electronics, vol. 16, no. 3, pp. 347-355, Mar 1980.

[31] D. Melati, F. Morichetti, and A. Melloni, "A unified approach for radiative losses and backscattering in optical waveguides," Journal of Optics, vol. 16, no. 5, p. 055502, 2014.

[32] P. Spano, S. Piazzolla, and M. Tamburrini, "Theory of noise in semiconductor lasers in the presence of optical feedback," IEEE Journal of Quantum Electronics, vol. 20, no. 4, pp. 350-357, Apr 1984.

[33] S. Latkowski, A. Hänsel, N. Bhattacharya, T. de Vries, L. Augustin, K. Williams, M. Smit, and E. Bente, "Novel widely tunable monolithically integrated laser source," IEEE Photonics Journal, vol. 7, no. 6, pp. 1-9, Dec 2015.

Theodorus T. M. van Schaijk (M'15) received the B.Sc. and M.Sc. degrees in electrical engineering from the Eindhoven University of Technology, Eindhoven, The Netherlands in 2012 and 2014, respectively. Since 2014 he has been pursuing the Ph.D. degree in the Photonic Integration group at Eindhoven University of Technology. His interests include the behaviour of lasers on generic III-V platforms and especially their feedback sensitivity.

Daan Lenstra (Amsterdam, The Netherlands, 1947) received the M.Sc. degree in theoretical physics from the University of Groningen and the Ph.D. degree from Delft University of Technology. Since 1979 he researched topics in quantum electronics, laser physics and condensed matter physics. Since 1991 he held chairs at the Vrije Universiteit in Amsterdam, Eindhoven University of Technology and Delft University of Technology. Presently, Daan Lenstra is full professor in the Photonic Integration group, Eindhoven University of Technology.

Erwin A. J. M. Bente (M'01) received the M.Sc. degree in physics and the Ph.D. degree from Vrije Universiteit, Amsterdam, The Netherlands, in 1983 and 1989, respectively. From 1988 to 1994, he was with Urenco Nederland B.V. and led a research group on laser isotope separation. From 1994 to 1996 , he was a Researcher with Vrije Universiteit, where he worked on solid-state coherent light sources and isotope separation of stable isotopes. He was a ResearchTeam Leader at the Institute of Photonics, University of Strathclyde, Glasgow, U.K., and was involved in high-power diode-pumped solid-state lasers, passive mode-locking, and femtosecond laser machining. Since 2001, he has been an Associate Professor in the Photonic Integration group, department of Electrical Engineering, Eindhoven University of Technology, Eindhoven, The Netherlands, where he is involved in integrated semiconductor laser systems.

Kevin A. Williams received his received the B.Eng. degree from the University of Sheffield, Sheffield, U.K., and the PhD degree from the University of Bath, U.K., in 1995. His research interests are in the area of integrated photonic circuits. He was awarded a Royal Society University research fellowship at the University of Bristol, Bristol, U.K in 1996. He moved to the University of Cambridge, Cambridge, U.K., in 2001 and was elected Fellow at Churchil College. In 2006, he was awarded a European Commission Marie Curie Chair at the Eindhoven University of Technology, The Netherlands. In 2011, he received a Vici award from the Netherlands Organization for Scientific Research (NWO). Kevin is chair of the Photonic Integration research group at Eindhoven University of Technology. 